\title{
Black hole formation preceded by a SN explosion or not: evidence from Galactic chemical evolution
}

\author{
Erwin De Donder and Dany Vanbeveren \\ Astrofysisch Instituut, Vrije Universiteit Brussel, \\ Pleinlaan 2, B-1050 Brussel, België
}

\begin{abstract}
We derive a constraint on direct black hole formation from the evolution of $[\mathrm{O} / \mathrm{Fe}]$ as function of time, during the early evolution of the Galaxy in the solar neighbourhood. Since oxygen is dominantly produced by massive stars, the evolution of $[\mathrm{O} / \mathrm{Fe}]$ is an indirect observable signature of the death of massive stars. We use a detailed Galactic code that computes as function of time the chemical composition of the interstellar gas, out of which successive generations of stars are formed. From our simulations we conclude, that to fit simultaneously the observed $[\mathrm{O} / \mathrm{Fe}]$ evolution and other observational constraints: $(i)$ all massive stars (single or binary) with $M>40 \mathrm{M}_{\odot}$ should form massive black holes with the ejection of at least $7 \mathrm{M}_{\odot}$ of oxygen and little carbon and iron; and (ii) mass loss by stellar wind during the Luminous Blue Variable phase and/or helium burning phase is likely to be metallicity dependent.
\end{abstract}

Black holes (BH) and neutron stars (NS) are the fossil cores of a massive star. While the formation of a NS always goes together with a supernova (SN) outburst, this is not clear for $\mathrm{BH}$ formation. Two possibilities exist: the core collapses directly into a BH without a SN explosion or mass ejection, or passes through an intermediate NS stage with some matter ejection, whereafter the NS turns into a BH by fall back or a phase transition during cooling. From theory, direct $\mathrm{BH}$ formation is predicted to occur above $40 \mathrm{M}_{\odot}$ and delayed $\mathrm{BH}$ formation with a SN outburst in the range $25-40 \mathrm{M}_{\odot}$ (Fryer 1999). In close binaries $\mathrm{BH}$ formation is expected to start at higher initial masses, because of enhanced mass loss by Roche lobe overflow (RLOF) that leads to the formation of smaller core masses. On the observational side, the high abundance of $\alpha$-elements in the atmosphere of the companion star in the BH X-ray binary Nova Sco has been considered as evidence for $\mathrm{BH}$ formation with matter ejection (Israelian et al. 1999). Also the observed superluminous SNe SN 1998bw, SN 1997ef and SN 1998ey are believed to be SN explosions with BH formation (Nakamura et al. 2001).

Adopting the two infall models of Chiappini et al. 1997 and following in detail the evolution of single and binary stars, we computed the evolution of the $[\mathrm{O} / \mathrm{Fe}]$ ratio as function of time for different assumptions on massive $\mathrm{BH}$ formation above $40 \mathrm{M}_{\odot}$. A detailed description of the Galactic code has been given in De Donder \& Vanbeveren (2002). Our computations are summarised in Figure 1. We conclude that if all stars above $40 \mathrm{M}_{\odot}$ collapse directly into a $\mathrm{BH}$ without matter ejection $(=\operatorname{sim} 1)$, the predicted $[\mathrm{O} / \mathrm{Fe}]$ ratio for $[\mathrm{Fe} / \mathrm{H}]<-1$ is an underlimit to the observations (Gratton et al. 1996; Israelian et al. 1998; 


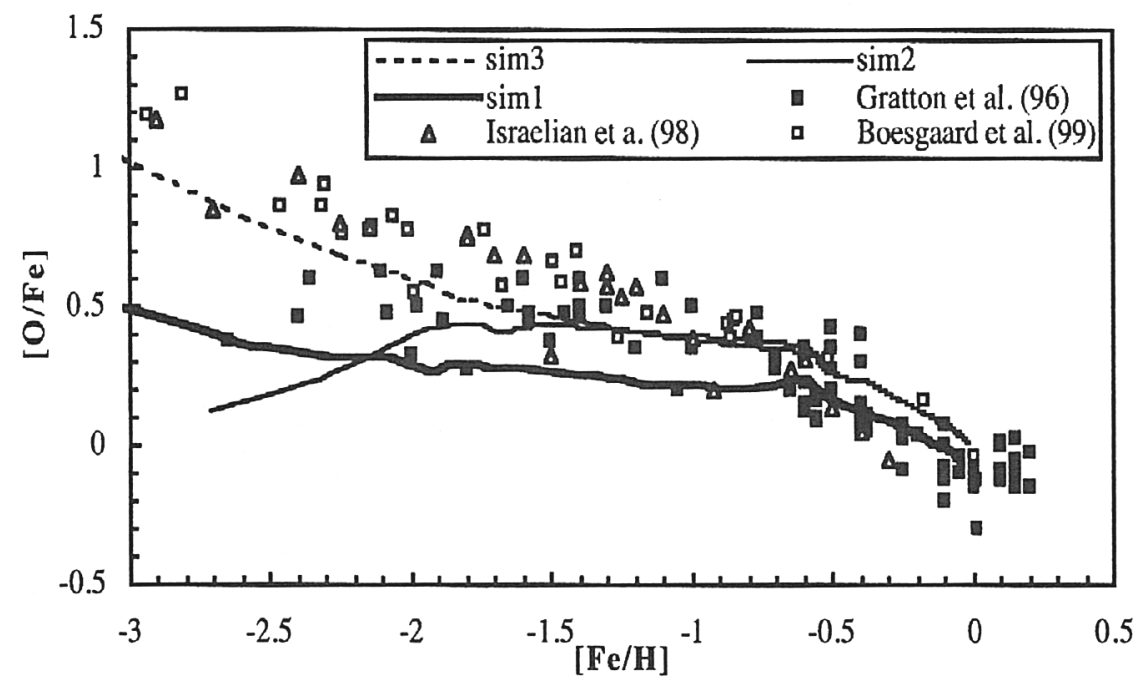

Figure 1. The predicted $[\mathrm{O} / \mathrm{Fe}]$ evolution compared with the observations.

Boesgaard et al. 1999). Much better agreement is found when large amounts of oxygen (at least $7 \mathrm{M}_{\odot}$ ) and few carbon and iron is ejected by stars above $40 \mathrm{M}_{\odot}$ (=sim3). In the hypernova model suggested by Nakamura et al. (2001), large amounts of iron are ejected to explain the light curve of the hypernova SN 1998bw. However, applying this model to stars initially more massive than $40 \mathrm{M}_{\odot}$ gives a too low $[\mathrm{O} / \mathrm{Fe}]$ ratio $(=\operatorname{sim} 2)$. The ejection of small amounts of iron may fit with the picture, that, due to inefficient mass loss by stellar wind (SW) at low metallicties, larger envelope masses are formed, and by consequence the main part of the inner region, where explosive silicon burning takes place, falls back during the explosion and goes into the $\mathrm{BH}$. Furthermore, we find that, if more than $1 \mathrm{M}_{\odot}$ of carbon is expelled, the $[\mathrm{C} / \mathrm{Fe}]$ ratio rises above the upperlimit on the observed [C/Fe] ratio during the early phases. All this requires that the bulk of ejected material must be oxygen, which implies that the formed massive CO core must be mainly composed of oxygen. This situation prevails when mass loss by SW during the LBV phase and/or helium burning phase is small. At low metallicities this may indicate that SW mass loss during the LBV and/or WR phase is metallicity-dependent.

\section{References}

Boesgaard, A.M., King, J.R., Deliyannis, C.P., Vogt, S.S. 1999, AJ 118, 2542

Chiappini, C., Matteucci, F., Gratton, R. 1997, ApJ 477, 765

De Donder, E., Vanbeveren, D. 2002, New Astron. 7, 55

Fryer, C. 1999, ApJ 522, 413

Gratton, R., Carretta, E., Matteucci, F., Sneden, C. 1996, in: H. Morrison \& A. Sarajedini (eds.), The Formation of the Galactic Halo, ASP-CS 92, 307

Israelian, G., García López, R.J., Rebolo, R. 1998, ApJ 507, 805

Israelian, G., Rebolo, R., Basri, G., Casares, J., Martín, E.L. 1999, Nature 401, 142

Nakamura, T., Umeda, H., Iwamoto, K., et al. 2001, ApJ 555, 880 